LUCAS CIIAMIONNIERE ON MASSAGE AND MOBLISATION IN THE TREATMEXT OF FKACIULES.'

In a monograph with the above title the author sets forth that which he has previously stated in the fournal de meteine et de chiru'sie pratique.

The author has used massage in the treatment of fractures for a number of years, and in I $S S 6$ in a memoir presented to the Soicte dichir. uricic he citcd to cases of fracture of the radius and fibula treated by this method.

The few motions and the slight kneading which M. Bourget used in the treatment of the fractures of the lower end of the radius can not be considered as massage. Onis in the fracture of the patella Mretzger and Tilanus advised rcgular massage and early walking, and this ac. cording to the writer is wrong, as fracture of the patella is one of the few fractures vilich should not be treated by massage.

It is well known that a certain amount of deformity does not interfere with the regular functions of the limb, provided the muscles and articulations are intact. But never mind how straight the rcpaired bone may be, or how slightly apparent the callus, the stiffncss of the muscles and joints is the cause of the nost marked functional disturbances, and it is to obviate this that the Championniere recommends his nethod.

T'he reason that massage has been unsuccessful in the hands of many surgeons is that it has been used too vigorously or too much violence has been used. Mr. Clampionniere divides massage in tour classes:

I. Inmediate massage. This is the most perfect way of applying the method. 'l'his is used where the chances of secondary displacement are slight, generally fractures around jounts. The fractures most suited for this treatment are lower part of radius and fibula, partial fractures

Juurnal de Mledicine et de Chirurgie Practique, December, $1 S S g$ 
of the elbow, head of humerus. and certain fractures of the cundyles of the femurs, and the supra-malleolar fractures without tendency to displacement. Here no immobilisation is used. It is only the tendency to displacement which should stop all immmediate manourres.

2. In the sarise fractures when the tendency to displacement is marked it is best to miassage the limb before placing it in an apparatus this applies particularly to fractures of the wrist, where there is great mobility, and for supra-malleolar fracture.

3. In a ccrtain class of fractures where thcre is much mobility without much tendency to displacement, such as arm, forearm and leg. The splints are applied, and removed in two or three days, when they are taken down and the parts are masseed, then a daily massage is made followed by replacing the splints.

4. Those cases of fracture where absolute immobility is rcquired for some period of time.

In these cases the motion of the fragments is vcry great, but rapid mobilisation is a necessity, here absolute immobility of the part for sonse days, and soon as the callus is sufficient, the apparatus is reinoved, and massage applied. To this class of cases belongs specially fractures of the upper and lower part of the lumerus.

There are three forms of movement eniployed in the massage of a fracture.

(I) Morements of exploration; (2) movements belonging properly to massage; (3) movements made in the surrounding and even in the affected joints.

1. Movements of Exploration.-As soon as a fracture is diagnosed, It is necessary to find out which motions can be gone through by the limb without causing any pain, and of what amount of motion the neighboring articulations are capablc without causing pain, and what regions can be pressed or rubbed. During this exploration the limb inust be immobilised, and the point of fracture held by the hand. The limb during this investigation may be placed on a sandbag. The author severely condemns that which he calls the barbarous praclicc of looking for crepitation.

2. diassage Propir.-The seat of fracture having been ascertained, 
which is a capital point, for it must not be subjected to direct pressure, and the limb having been fixed, and the point of fracture immobilised, the massage is begun. Pressure is made with the hand, and is perpen. dicular to the axis of the limb. With this pressure is combined a sliding of the hand along the limb. This sliding of the hand must always be in the direction of the axis of the limb, and following the direction of the venous return. The pressure and gliding of the hand must always be in one direction, never up and then down. Besides these two movements, a third is often used, it consists of circular pressure, a sort of grinding rotary movement exercised with the palm of the hand. This is to be used wherever there is much tumefaction, and any localized exudation.

The part of the land most used in massage of fractures is the thumb, after that, when more force is required the palm of the hand.

Pétrissage of the muscles should not be performed as it is too rough and painful.

Massage must not cause pain, it must relieve it. The manauvers must be progressive; the first mu-t be extremely gentle.

The length of a seance is at least fifteen minutes. It is important that the skin of the operator, and of the one operated on. should be extremely soft, and supple, the best substance to use to facilitate the motion is perfectly pure oil.

Moiements to be made by the fractured limb, and by the neighborin! farts.-When the seance of massage has been ended, all the small jounts of the neighborliood mist be worked, as well as the large articulations, these motions should cause no pain or displacement of the fragments.

The movements are two fold, active and passive, but should not be too extensive. The massage should not be practised oftener than once in twenty-four hours, and after the first week $t$ is not necessary to apply massage daily, every two or three days will suffice. The first seance of massage should take place as soon as possible after the fracture has occurred. Except in those cases where there is much tendency to displacement no splint should be applied after the massage. Dr. Lucas Championniere absolutely condemns the use of an elastic bandage after 
a fracture, as has been advised by Oberst of Halle, Vide Axisus of Surgeky, December, $1 S S 8$. When there is no tendency to displacement a simple flannel bandage is a sufficient dressing.

Action of Massage.-The first result is to cause a disappearance of the pain. then, by favoring absorption, it causes the tension due to extravasation to disappear. MLoreover, the blood.clots, which act as irritants, are displaced, broken up and more easily absorbed. The swelling, under massage, disappears with great raptdity. Also under massage the slight muscular ruptures heal readily. The only contraindication to massage lies in the extreme mobility of the fragncnts. If, although there is great mobility of the fragnonts, they can be tcmpo. rarily immobilized, massage should be practiced, but if the mobility of the Iragments renders a permanent deformity liable, massage should be abandoned.

\section{Special Fractures.}

Fracture of the Radius at Its Loiier E.vtremity is the fracture the best suited to massage. If the deformity is very marked and shows much tendency to return, after the first seance of massage a splint should be applied for five or eight days, and then the treatment begun anew; otherwise a daily massage, and no splint nced be applied afterwards. Massage in these cases should be applied to the wrist joint, to the fingcrs and to the muscles as far as the elbow joint, care being taken to avoid the point of fracture. This same treatment is applicable to fracture of any part of the bone.

Fracture of the Fibula.-The only contra-indication to the massage treatment here is not the lateral mobility of the fragments, but the tendency to eversion of the foot, and this is easily remedied. The frac. tures around the malleoli give the best rcsult. Ordinarily afier three weeks the patient is cured.

Fractures of Both Malleoli give excellent results with this form of treatment, as there is much pain and considerable swelling, both of which subside readily under the above iescribed form of treatment. If there be much tendency to displacement, the leg should be immediately masséed, then put up in an immovable dressing for eight or ten 
days, and afterwards taken down and massied daily and replaced in the splint. In these cases the massage should be carried on as high as the thigh.

Fractures of the Head of the Tibia.-In these the same course should be pursued, only the seance must be more prolonged.

Fractures of Both Bones of the Forearm are to be treated by what Lucas Championniere calls the mixed method, that is, massage, immobility in a splint for eight or ten days, when it is left off and massage again used.

Fractures of the E/boni.-Where there is no deformity no apparatus should be used. Most cases are those in children where there is fracture of the lumerus which simulates a backward dislocation; here the tendency of a return of the deformity is so great that an immovable apparatus should be applied after the first seance and left on from is to so days.

Fracture of the Olecratton. - In these cases. like in fracture of the patella, the writer adrocates cutting down and suturing the bone, and later on, massage.

Fracture of the Ufper End of the Humerus. - Fracture of anatomical neck without displacement must be massied immediately and the seance will have to be prolonged; the pain, which is marked, will persist for some time; the whole of the shoulder should be included in the trentment.

Fracture of surgical neck with displacement of the lower fragment should be brought in proper place, and mixed method used if there is much mobility or tendency to the return of the deformity. The seance in these cases must be very long. If necessary, administer chloroform for the first sitting, and do the massage while the patient is anzesthetized.

Fracture of the Neck of the Femur.-Massage; patient to get out of bed as soon as possible; care taken during the massage not to exert much pressure in the inguinal region or Scarpa's space.

Fracture of Shaft of Femur.-Massage and the use of Hennequin's spliut, or massage combined with cxtension.

Fracturi of thi Patella.-Same treatment advocated as for that of 
olecranon; suture, then massage; massage alone in this fracture gives bad results.

For the past five years the author has treated all the cases of fracture, both in hospitals and private practice, by the method above outlined, and claims only good results. He employed massage in the treatment of fractures of the lower extremity of the radius some fifteen years ago, and claims the priority for this form of treatment.

F. C. Hussos.

\section{JAFFE ON THE THERAPY OF HABITUAL SCOLIOSIS.}

In a recent number of Volkmann's Sammlung klinischer Voringge, Dr. Max Jaffè, of Posen, discusses the myopathic theory of scoliosis, and the therapy based thereon.

He does not think that the curvature is produced by antagonism in muscular action (the muscles on the concive side of the curve being contracted, while those on the other side are in a condition of extension.) He states that the position in which, according to myopathists, the muscles contract in order to naintain their posture, are not those in which we are accustomed to employ muscular action, they are positions of rest, maintained without any muscular contraction.

Differences in muscular development on both sides are never observed in commencing scoliosis, and the results of autopsies on which myopathists base their theories only exist in chronic cases with extreme deformity.

The therapeutics based on the myopathic theory, unilateral massage and exercise, are of no service whatsoever, and are not comparable to the symmetrical development of the entire muscles of the back. Whatever successes have been obtained by myopaths depend on the fact that it is impossible to act upon special muscular groups without acting on others as well.

Therefore it should be endeavored to secure a uniform and symıetrical development of the spinal muscles. This treatment satisfies the physician that in scoliosis badly dereloped and atrophied muscles are present in all portions of the back, and of the correctness of Meyer $\mathbb{S}$ 